\title{
LA RIABILITAZIONE CON TECNICHE COGNITIVO-COMPORTAMENTALI NEL PAZIENTE CON SINDROME DI ASPERGER PER UNA TRANSIZIONE NELL'ETÀ ADULTA
}

\author{
Dott. Perfetto Viviana, Dott. D’Ambrosio Antonio
}

KEYWORDS: Asperger, riabilitazione psichiatrica

\section{ABSTRACT}

$\grave{E}$ stata ormai ampiamente dimostrata l'efficacia delle tecniche cognitivo - comportamentali in moltissimi disturbi di natura psichiatrica, ma ancora oggi le evidenze lasciano una zona d'ombra sull'applicazione di questi interventi ai disturbi generalizzati dello sviluppo. Questo studio si propone quindi di individuare e trattare alcune delle problematiche tipicamente presenti in questi soggetti, riconducibili alle aree del contatto visivo, della risposta al nome, e del linguaggio espressivo e recettivo.

Il campione è rappresentato da dieci pazienti adolescenti con diagnosi di Sindrome di Asperger, afferenti al PAIDèS (polo aziendale di intervento sui disturbi generalizzati dello sviluppo) dell'ASL Napoli 2. Per ogni paziente, valutato utilizzando ADI-R e ADOS, sono stati individuati tre obiettivi mediante la compilazione di un'apposita check list; lo scopo dello studio è quello di stimare l'apprendimento di tali abilità su breve termine, verificando se l'utilizzo di tecniche cognitivo - comportamentali rappresenti un metodo più rapido per migliorare alcuni aspetti del comportamento e dell'interazione in questi soggetti. Gli obiettivi sono stati concordati con i genitori dei pazienti, in modo tale da evidenziare gli aspetti maggiormente problematici ed individuare target terapeutici raggiungibili in un breve periodo di tempo.

L'ADI-R e l'ADOS si sono rivelati ottimi strumenti di valutazione, completi e complementari, che consentono una visione ampia del passato e del presente dei pazienti coinvolti; la check list è stata invece utilizzata durante i primi incontri per individuare gli obiettivi su cui impostare il lavoro successivo, ed alla fine dello studio per poterne valutare l'effettivo raggiungimento. E' previsto, infine, un follow up a 6 mesi con ADOS e check list per valutare il mantenimento nel tempo dei risultati ottenuti.

\section{INTRODUZIONE}

Troppo spesso la Sindrome di Asperger viene considerata una problematica legata esclusivamente all'età evolutiva, con interventi e cure che si concentrano durante l'infanzia, e spariscono quasi del tutto in età adulta. I disturbi di spettro autistico, di cui tale sindrome fa parte, sono invece condizioni persistenti durante la crescita, che accompagnano l'individuo in ogni fase della vita, e hanno quindi bisogno di interventi appositi anche in età adulta, quando il paziente viene ancora oggi spesso "dimenticato".

Il bambino autistico diventerà un adulto autistico, ed è fondamentale in quest'ottica prevedere un piano di trattamento che possa considerare l'evoluzione del paziente nella sua totalità, adattandosi a bisogni e cambiamenti del soggetto e facendo leva su un indispensabile lavoro d'equipe, vera risorsa di una riabilitazione efficace.

Questo studio si propone, su queste basi, di fornire un valido strumento di lavoro che possa servire da stimolo e da ispirazione per una maggiore attenzione verso il trattamento continuativo delle sindromi di spettro autistico.

\section{LA SINDROME DI ASPERGER CRITERI DIAGNOSTICI}

La diagnosi di Sindrome di Asperger viene attualmente formulata facendo riferimento ai criteri del Manuale Diagnostico e Statistico dei disturbi mentali
(DSM-IV), in cui si colloca tra i Disturbi Pervasivi dello Sviluppo. Di seguito sono riassunti i criteri diagnostici utilizzati dal DSM-IV e dall'ICD-10.

\section{Disturbo di asperger - dsm-iv}

1. Compromissione qualitativa nell'interazione sociale, come manifestato da almeno due dei seguenti:

- $\quad$ marcata compromissione nell'uso di diversi comportamenti non verbali come lo sguardo diretto, l'espressione mimica, le posture corporee ed i gesti che regolano l'interazione sociale;

- incapacità di sviluppare relazioni con i coetanei adeguate al livello di sviluppo;

- mancanza di ricerca spontanea della condivisione di gioie, interessi o obiettivi con altre persone;

- mancanza di reciprocità sociale o emotiva.

2. B. Modalità di comportamento, interessi e attività ristretti, ripetitivi e stereotipati, come manifestato da almeno uno dei seguenti:

- dedizione assorbente ad uno o più tipi di interessi stereotipati e ristretti, che risultano anomali o per intensità o per focalizzazione;

- $\quad$ sottomissione del tutto rigida ad inutili abitudini o rituali specifici; 
- manierismi motori stereotipati e ripetitivi;

- $\quad$ persistente ed eccessivo interesse per parti di oggetti.

3. C. L'anomalia causa compromissione clinicamente significativa dell'area sociale, lavorativa o di altre aree importanti del funzionamento.

4. D. Non vi è un ritardo del linguaggio clinicamente significativo.

5. E. Non vi è un ritardo clinicamente significativo dello sviluppo cognitivo o dello sviluppo di capacità di autoaccudimento adeguate all'età, del comportamento adattivo (tranne che dell'interazione sociale) e della curiosità per l'ambiente nella fanciullezza.

6. F. Non risultano soddisfatti i criteri per un altro specifico Disturbo pervasivo dello Sviluppo o per la Schizofrenia.

\section{Sindrome di asperger - icd-10}

1. Non è presente un ritardo generale clinicamente significativo nell'espressione o produzione del linguaggio o nello sviluppo cognitivo. La diagnosi richiede che l'uso di singole parole si sia sviluppato entro i primi due anni di età e che l'uso di frasi a valenza comunicativa si sia sviluppato entro i primi tre anni di età. L'autosufficienza, l'adattamento all'ambiente e la curiosità per l'ambiente nei primi tre anni debbono essere di un livello adeguato al normale sviluppo intellettivo. Tuttavia, gli stadi dello sviluppo motorio possono essere in qualche misura ritardati ed è frequente una certa goffaggine motoria (sebbene non sia una caratteristica diagnostica necessaria). Sono frequenti, ma non sono necessarie per la diagnosi, particolari abilità isolate, spesso connesse ad interessi normali.

2. Sono presenti compromissioni qualitative dell'interazione sociale.

3. Il soggetto presenta un interesse inusualmente intenso e circoscritto o modelli di comportamento, interessi e attività limitati, stereotipati e ripetitivi.

4. Il quadro non è attribuibile ad altri tipi di sindromi da alterazione globale dello sviluppo psicologico, alla schizofrenia simplex, alla sindrome schizotipica, alla sindrome ossessivo - compulsiva, al disturbo di personalità anancastico, al disturbo reattivo e disinibito dell'attaccamento dell'infanzia.

\section{LA TERAPIA COGNITIVO}

\section{COMPORTAMENTALE}

La terapia cognitivo - comportamentale (Cognitive Behavior Therapy, CBT), per definizione, insegna alle persone a monitorare i propri pensieri e le proprie percezioni al fine di renderle più consapevoli dei propri errori interpretativi; tutto ciò richiede un certo livello di sviluppo cognitivo e verbale, quantificato in una revisione sistematica come un minimo di QI verbale pari a 69, quindi è rivolta al sottogruppo di soggetti con autismo ad alto funzionamento o Sindrome di Asperger. Sono disponibili due studi sulla CBT in bambini con disturbi dello spettro autistico: una revisione sistematica che indaga l'efficacia della CBT nel trattamento dei disturbi d'ansia e un singolo RCT $(n=45)$ che indaga l'efficacia della CBT nel trattamento delle crisi di rabbia.
La revisione sistematica include 9 studi $(n=110)$; i partecipanti sono ragazzi con sindrome di Asperger o autismo ad alto funzionamento, con un'età compresa tra i 9 ed i 23 anni, in maggioranza maschi (oltre il 60\%), che soffrono di comorbidità con disturbi d'ansia. L'intervento di CBT ha in media una durata di 4 mesi (da 6 settimane a 6 mesi), è somministrato per circa 12 sessioni (da 6 a 16) della durata media di 1,25 ore (1-2 ore); è erogato da terapeuti formati e con esperienza nel campo. I risultati degli studi inclusi sono coerenti nel riportare un miglioramento significativo dei sintomi d'ansia nel gruppo che riceve l'intervento di CBT; inoltre in alcuni studi è riportata una riduzione dell'entità dei sintomi d'ansia tale per cui i soggetti alla conclusione del trattamento non soddisfano più i criteri per la diagnosi di comorbidità con il disturbo d'ansia.

L'RCT, invece, confronta l'efficacia di un intervento di CBT altamente strutturato, rivolto a bambini con sindrome di Asperger e ai loro genitori e finalizzato a migliorare la capacità di gestione della rabbia, con nessun trattamento. Il campione $(n=45)$ è composto da bambini di 11 anni, per la maggioranza maschi $(n=43)$, che nel 44\% dei casi presenta comorbidità con ADHD. L'intervento di CBT, della durata di 6 settimane, è erogato individualmente in sessioni di due ore a settimana; anche i genitori ricevono alcune ore di trattamento di gruppo. L'intervento è finalizzato ad operare strategie alternative per risolvere le situazioni in cui il bambino manifesta crisi di rabbia.

I miglioramenti riportati dai genitori riguardano una maggiore capacità di gestione delle crisi dei figli; nei bambini, invece, si rileva una riduzione di frequenza ed intensità delle crisi di rabbia, una maggiore tolleranza alla frustrazione ed un migliore rapporto con l'autorità (genitore ed insegnante).

In conclusione, è consigliato l'uso della terapia cognitivo - comportamentale per il trattamento della comorbidità con i disturbi d'ansia nei bambini con sindrome di Asperger o autismo ad alto funzionamento; tale approccio, inoltre, può essere utile anche nel migliorare le capacità di gestione della rabbia.

\section{Protocollo sperimentale}

Questo studio ha valutato l'apprendimento specifico per obiettivi in un breve arco temporale (sei mesi) in un gruppo di dieci pazienti con Sindrome di Asperger (DSM-IV) afferenti al PAIDèS (polo aziendale di intervento sui disturbi generalizzati dello sviluppo) dell'ASL Napoli 2.

\section{IMPLEMENTAZIONE DEL PROGETTO}

Allo scopo di valutare gli obiettivi, è stata utilizzata un'apposita check list somministrata individualmente ad ogni partecipante dopo un periodo di osservazione e valutazione. Le aree che si sono evidenziate come particolarmente problematiche (tipiche del disturbo) riguardano:

\section{Contatto visivo; \\ 2. Risposta al nome; \\ 3. Linguaggio espressivo; \\ 4. Linguaggio recettivo.}

Per ogni partecipante si sono individuati uno o più obiettivi finali su cui dover lavorare, appartenenti alle aree sopra citate, concordandoli con i genitori dei pazienti e pianificando una verifica dei risultati a breve termine dell'intervento su queste aree. 


\section{METODOLOGIA}

Per ogni partecipante sono stati effettuati:

1. Fase di valutazione Quattro/cinque incontri, così suddivisi:

a. Un incontro della durata di circa un'ora e mezza con i genitori per la compilazione dell'ADI-R, un'intervista semi- strutturata.

b. Un incontro di un'ora con il paziente per lo svolgimento dell'ADOS, un modulo che si basa sull'osservazione del soggetto nel corso di alcune attività appositamente strutturate.

c. Due/tre incontri di valutazione informale con il paziente, durante i quali sono state raccolte le informazioni necessarie per compilare la check list ed individuare gli obiettivi da proporre alla famiglia.

2. Definizione degli obiettivi Un incontro con i genitori per concordare gli obiettivi di maggior rilievo su cui poter basare il lavoro successivo.

3. Fase di trattamento Quattro/cinque incontri con il paziente finalizzati al raggiungimento degli obiettivi stabiliti attraverso l'utilizzo di tecniche cognitivo - comportamentali.

4. Fase di verifica ADI-R, ADOS e check list sono stati somministrati ad ogni partecipante ad inizio trattamento, e solo la check list è stata ri-compilata alla fine del ciclo di incontri per valutare l'effettivo raggiungimento degli obiettivi proposti.

\section{STRUMENTI}

Gli strumenti di valutazione utilizzati sono stati i seguenti:

- Autism Diagnostic Interview - Revised (ADI-R), un'intervista finalizzata ad ottenere una gamma completa di informazioni per la valutazione dei disturbi di spettro autistico. L'intervista è rivolta ai genitori o agli educatori di soggetti dalla prima infanzia all'età adulta, e si focalizza sull'osservazione sistematica e standardizzata di comportamenti che raramente vengono riscontrati in soggetti non clinici. L'ADI-R approfondisce principalmente le tre aree del funzionamento che vengono indicate di importanza diagnostica secondo i criteri dell'ICD-10 e del DSM-IV: linguaggio e comunicazione, interazione sociale reciproca, comportamenti stereotipati ed interessi ristretti.

- Autism Diagnostic Observation Schedule (ADOS), un protocollo di osservazione semistrutturato, standardizzato per la valutazione della comunicazione, della reciprocità sociale e del gioco simbolico in soggetti con disturbi dello spettro autistico. L'ADOS consiste in una serie prestabilita di attività durante le quali il somministratore ha la possibilità di rilevare i comportamenti maggiormente evidenti in questo tipo di disturbi. Le attività che vengono proposte sono tutte studiate per spingere il soggetto a mettere in atto comportamenti di tipo comunicativo, sociale, e che riflettano la sua competenza simbolica: tali attività, che vanno dal giocare con le bolle di sapone nel modulo 1 alla conversazione sulla propria vita sociale nel modulo 4, non sono auto-finalizzate, ma hanno lo scopo di creare un'interazione, nel corso della quale emerga lo stile relazionale e comunicativo del soggetto.
- Check list appositamente adattata per la definizione di obiettivi riguardanti le aree del linguaggio espressivo e recettivo, del contatto visivo e della risposta al nome. Questa scheda è stata realizzata sulla base della check list elaborata da Bridget Taylor in "Behavioural intervention for young children with autism: a manual for parents and professionals publisher" [1996].

Per quanto riguarda gli strumenti di intervento, sono state invece utilizzate:

- Storie sociali

- Schede di conversazione

- Schede delle emozioni

\section{A. LE STORIE SOCIALI}

Una storia sociale è un breve racconto, costruito seguendo una struttura ben definita, che serve a descrivere in modo oggettivo un'abilità, un evento, un concetto o una situazione sociale.

Ogni storia sociale fornisce al destinatario informazioni chiare, concise ed accurate su quello che sta accadendo in una specifica situazione sociale, delineando la causa dell'evento ed un'eventuale risposta adeguata. Questi approcci cercano di fornire al destinatario delle risposte a domande chiave riguardanti una situazione sociale problematica, chiarendo quindi:

- Cosa sta succedendo?

- Chi sta facendo cosa?

- Perché succede?

- Quale regola culturale, istituzionale o individuale sta alla base dei comportamenti delle persone?

- Quali sono le tipiche risposte accettabili socialmente che il ragazzo può dare nella situazione specifica?

La storia sociale, rispondendo in modo chiaro a queste domande, rende esplicito ciò che i ragazzi con un normale sviluppo capiscono da soli grazie ai messaggi sociali espliciti o impliciti contenuti nell'esperienza di tutti i giorni. I soggetti con disturbi dello spettro autistico spesso invece non riescono ad utilizzare l'intuito e l'imitazione per comprendere cosa accade nelle situazioni sociali e quale risposta adeguata poter mettere in atto.

\section{B. LE SCHEDE DI CONVERSAZIONE}

Tali strumenti sono utili in quanto danno ai pazienti delle regole precise su cui basare ogni tipo di conversazione; in aggiunta, sono state utilizzate varie schede che fungono da veri e propri "suggerimenti di conversazione" per i ragazzi, consigliando possibili domande, commenti e risposte su cui poter costruire un dialogo.

\section{C. LE SCHEDE DELLE EMOZIONI}

Molte emozioni sono difficili da insegnare perché non sono facilmente definibili. Per questo motivo, è importante che il paziente possa collegarle direttamente alla sua esperienza per renderle il più possibile esplicite ed identificabili. Molti ragazzi con disturbi dello spettro autistico, pur sperimentando stati emotivi complessi come la delusione o la rabbia, non riescono a rispondere a domande come "Che cosa ti fa arrabbiare?" o "Quand'è che ti senti deluso?", oppure non riescono a riconoscere tale emozione nell'altro. 
Lo scopo di questi esercizi è quindi quello di insegnare al paziente le abilità necessarie per identificare, definire ed esprimere le emozioni fondamentali in modo appropriato.

Tale incapacità di percepire, descrivere e verbalizzare le proprie o le altrui emozioni è detta alessitimia.

\section{LA SELEZIONE DEI PAZIENTI}

I partecipanti sono stati selezionati rispettando i seguenti criteri di inclusione:

- Età adolescenziale o pre-adolescenziale

(10-17 anni);

- Diagnosi di Sindrome di Asperger;

La scelta dei dieci utenti tra la popolazione con le caratteristiche descritte è stata effettuata tramite un processo di randomizzazione semplice. Prima dell'inizio del progetto, ogni paziente, insieme ai genitori, ha incontrato gli operatori per un colloquio individuale orientato a sondare la motivazione, la disponibilità e i vissuti relativi all'idea di provare l'esperienza; in questa sede ogni genitore ha firmato il consenso informato.

Le figure professionali coinvolte nello studio sono: una sociologa, una logopedista, una terapista della neuro psicomotricità ed una terapista della riabilitazione psichiatrica.

\section{SCHEMATIZZAZIONE}

DEI RISULTATI $(\mathbf{N}=10)$

Abbiamo riportato i dati come percentuali di compromissione rispetto ai valori iniziali in quanto si tratta di valori espressi dalle rispettive scale in modo disomogeneo dal punto di vista quantitativo, avendo ognuna di esse differenti standard di riferimento.

La richiesta di aiuto ed il riconoscimento delle emo-

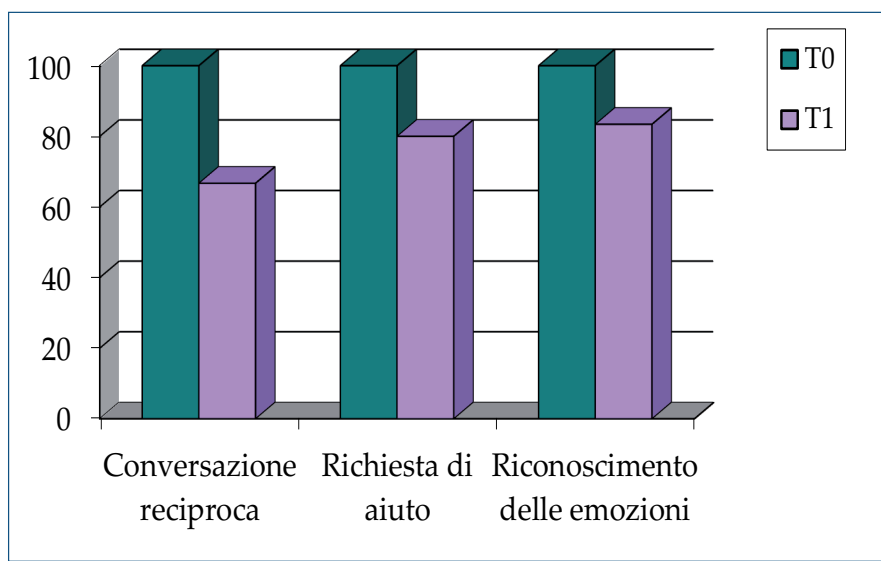

Fig. 1

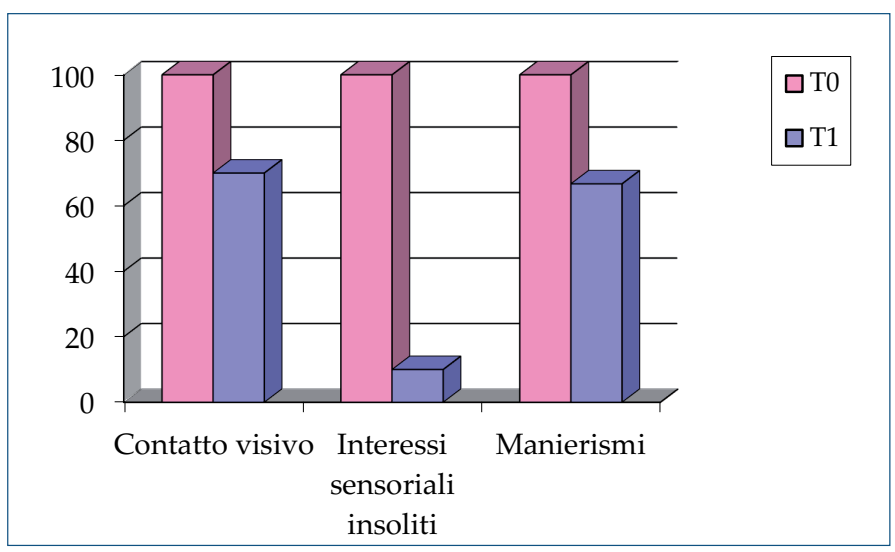

Fig. 2 zioni sono stati valutati attraverso la check list, che ha dei valori di funzionamento crescente che vanno da uno a dieci. É questo il motivo per cui questi due items sono gli unici ad avere un valore che aumenta da T0 a T1, mentre gli altri (appartenenti all'ADI-R o all'Ados) classificano il miglioramento in una scala decrescente.

\section{CONCLUSIONI}

I pazienti con diagnosi di Sindrome di Asperger selezionati per questo studio hanno risposto nel complesso in modo soddisfacente alle tecniche cognitivo - comportamentali utilizzate, con un miglioramento medio del $38,8 \%$. Nello specifico, si è descritta una riduzione della compromissione rilevata nella valutazione iniziale come segue:

- La compromissione dei ragazzi riguardante le abilità di conversazione si è ridotta in media del $37,2 \%$;

- La richiesta di aiuto è migliorata del $20 \%$ nei pazienti coinvolti;

- Il riconoscimento delle emozioni ha raggiunto una diminuzione media di compromissione del $16,6 \%$;

- $\quad$ Il contatto visivo è migliorato del $30 \%$;

- Il lavoro sui manierismi, infine, ha provocato nei pazienti coinvolti un miglioramento del 33,3\% .

Nel breve termine, le tecniche utilizzate sono state molto utili nel perseguire obiettivi vari e diversificati, generando una risposta positiva nei pazienti e nei loro familiari. In particolare le storie sociali hanno avuto molto consenso tra i ragazzi; probabilmente, questo è il risultato della peculiare comprensibilità e chiarezza delle storie sociali nel descrivere alcuni tipi di comportamenti e le rispettive risposte sociali. Questo risultato è stato sottolineato anche da uno studio recentissimo [Scheeren, de Rosnay et al., 2012] che ha evidenziato come le storie sociali siano particolarmente valide per la riabilitazione di bambini ed adolescenti con disturbi dello spettro autistico, garantendo un'efficacia a breve termine. In tale studio le storie sociali sono state utilizzate per far comprendere ai pazienti il significato del sarcasmo e per sfatare false credenze; nel presente studio è stato invece ampliato il loro utilizzo.

Il lavoro sulle emozioni ha mirato molto al coinvolgimento dei soggetti e, così come quello sulla conversazione, ha rilevato un'efficacia considerevole. Data la notevole compromissione dell'abilità di riconoscere e distinguere le emozioni, presente nei soggetti con Sindrome di Asperger, i risultati più difficili da ottenere sono stati quelli relativi alla paura e alla rabbia, mentre è risultato più semplice lavorare sulle emozioni positive (es. felicità). Questo è in linea con quanto descritto da un recente studio di Uljarevic ed Hamilton [2012], che ha evidenziato come l'emozione più difficilmente identificabile per tali soggetti sia la paura, mentre la più semplice sia la felicità.

Per quanto riguarda le abilità di conversazione, invece, uno studio del 2007 [Mackay, Knott \& Dunlop] ha organizzato un training comunicativo di gruppo, coinvolgendo bambini ed adolescenti con Sindrome di Asperger. Il nostro approccio si è mostrato efficace nell'incrementare il repertorio comunicativo dei pazienti coinvolti, con un riscontro positivo anche da parte delle impressioni dei familiari, che hanno 


\begin{tabular}{|l|l|l|}
\hline Obiettivo & T0 & T1 \\
\hline Conversazione reciproca & 2,2 & 1,4 \\
\hline Richiesta di aiuto & 4,6 & 6,6 \\
\hline Riconoscimento delle emozioni & 4 & 5,6 \\
\hline Contatto visivo & 1,8 & 1 \\
\hline Interessi sensoriali insoliti & 2 & 0 \\
\hline Manierismi & 2 & 1 \\
\hline
\end{tabular}

Tab.1

notato immediatamente i miglioramenti dei ragazzi. Ovviamente alcuni ragazzi avranno bisogno di lavorare ancora molto e di continuare ad essere seguiti in modo continuo nel tempo per poter interiorizzare alcuni comportamenti e comprendere fino in fondo alcune regole sociali, ma già nel breve termine $\mathrm{i}$ risultati sono stati soddisfacenti.
Interventi validi ed efficaci dovrebbero quindi essere svolti anche nell'individuo adulto, in cui non è detto che debba essere persa la speranza di un miglioramento. In questo senso, un lavoro di equipe svolto da professionisti appositamente formati e specializzati in tali disturbi può essere l'unica strada in grado di garantire un'ottica di continuità.

\section{REFERENCES}

1. American Psychiatric Association, Diagnostic and statistical manual of mental disorders - IV, (1994).

2. Freeman S., Dake L., Il linguaggio verbale nell'autismo. Strategie di insegnamento per bambini con disturbi dello spettro autistico. Ed. Erickson, (2007).

3. Gauss V.L., Cognitive behavioral therapy for adult with Asperger Syndrome. Guilford Press, (2007).

4. Lang R., Regester et al., Treatment of anxiety in autism spectrum disorders using cognitive behaviour therapy: A systematic review. Dev Neurorehabil, (2010).

5. Lord C., Rutter M., DiLavore P.C., Risi S., Autism diagnostic observation schedule. Giunti OS, (2005).

6. Mackay T., Knott F., Dunlop AW, Developing social interaction and understanding in individuals with autism spectrum disorder: a groupwork intervention. J Intellect Dev Disabil, (2007).

7. Organizzazione Mondiale della Sanità, International classification of diseases - 10, (1990).

8. Rutter M., Le Couteur A., Lord C., Autism diagnostic interview - Revised. Giunti OS, (2005).

9. Scheeren AM, de Rosnay M., Koot HM, Begeer S., Rethinking theory of mind in highfunctioning autism spectrum disorder. J Child Psychol Psychiatry, (2012).

10. Smith C., Storie sociali per l'autismo. Sviluppare le competenze interpersonali e le abilità sociali. Ed. Erickson, (2006).

11. Società Italiana di Neuropsichiatria dell'infanzia e dell'adolescenza, Linee guida per l'autismo, (2011).

12. Sofronoff K., Attwood T. et al., A randomized controlled trial of a cognitive behavioural intervention for anger management in children diagnosed with Asperger syndrome. J Autism Dev Disord, (2007).

13. Uljarevic M., Hamilton A., Recognition of Emotions in Autism: A Formal Meta-Analysis. J Autism Dev Disord, (2012).

14. White AH, Cognitive behavioural therapy in children with autistic spectrum disorder. STEER, (2004). 